\title{
Research and Practice on Surface Reconstruction by Region \\ DONG Tong
} Department of Mechanical Engineering, Dalian Vocational \& Technical College, Dalian, 116037 ,
China

65485484@qq.com

Keywords: Reverse engineering, region, mesh fitting

\begin{abstract}
In recent years, the method of parametric modeling has become an important direction of research in reverse engineering based on division of region, which is a more effective and accurate solution for surface reconstrution. This paper analyzed and compared many examples, summarized the influence of shape and accuracy by different division of region, and ensure the efficiency and accuracy of surface reconstrution.
\end{abstract}

\section{Introduction}

Surface reconstruction is a focus in reverse engineering, meshing fitting can get high-accuracy free-form surface, the degree of accuracy lies on the division of regions to a great extent. So, it is necessary to research on the problem of region division.

\section{Reverse Engineering}

Reverse engineering is used to describe the process of products design. The engineers commonly consider the process of products design is a process from nonexistence to pass into existence, the designer conceives the contour, capability, technical parameters of the products in brain, then, builds the 3D digital model, finally, put the model to the manufacturing process, complete the whole the cycle of design and manufacture. This is the process which is called forward design. Reverse engineering product design is the process from existence to nonexistence, to be short, Reverse engineering product design is the process of obtain the design data ( include design drawings or mathematical model ) based on the existed product model [1].

\section{Region}

Polygon mesh is foundation to reconstruct the real objects. In the process of inverse parameter modeling, After scaning the product, we get point - cloud data, which need to be handled to polygon mesh. Polygon mesh consist of triangular patch, include unit face, unit sideline, unit vertex, boundary [2], as shown in Figure 1.

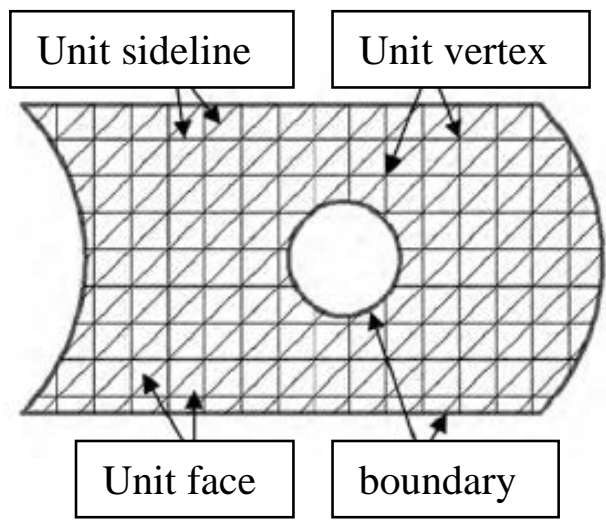

Fig. 1 polygon mesh

Region consist of unit faces, not include unit sideline and unit vertex, region is divided by curvature. When mesh fitting, region can avoid the influence of unit sideline and unit vertex, which 
can get more accurate surface. Divide region by curvature can make a distinction between different features, also can divide the same feature by different curvature, which can show the features better [3].

\section{Reverse Modeling Method Based on Division of Region}

Here, we discuss the process of the reverse modeling method based on division of region. First, divide the grid into different regions according to curvature, the model feature will show by the regions. Then, set up coordinate system on the basis of region, in order to draw profile of the section and project boundary profile [4], also, we need modify parameter value and add constraint to get accurate sketch, which will be stretched, revolved and swept to the model. The surface feature can be obtained by surface fitting. Finally, we can get the original model by Boolean operation of surfaces and models [5].

\section{Reverse Modeling Software}

Geomagic Design X software reconstruction the model based on division of region, which can divide different features or divide the same feature based on different curvature, which is good at set up irregular feature parametric model, fitting complicated surface accurately, in order to establish accurate parameterized CAD model. In Geomagic Design X software, mesh fitting is applied adequately. Mesh fitting, which take advantage of computer numerical operation, establish 3D surface based on mesh, give a high-performance modeling thought [6].

\section{Example}

Every complicated surface is composed of many simple surface, include quadric surface and free-form surface. Reconstruction the model can include many steps: first, fitting simple surface partly, then, surface transition, insection, cutting, edge blend etc, finally, sew the surfaces together.we need analyse the form of the sample piece, try to figure out the designer's intention, infer the type of the original surface, the way to reconstruction the model, which can help us divide the point cloud correctly.

As the example shown in Figure 2, the fish model is needed to be reconstruction. Firstly, need to divide the region, there are two plans, as shown in Figure 3, one plan divide the surface into two regions, the other plan divide the surface only one region. The result of mesh fitting is shown in Figure 4, then we can see that the continuity of surface which is fitted by two regions is not good, it is very difficult to transit the two surface. By this example, we can see that the number of the regions needed to be divided lies on continuity and accuracy, more regions can get more accurate, but the continuity is bad. So, we need to reduce the number of regions below the premise that assures accuracy.

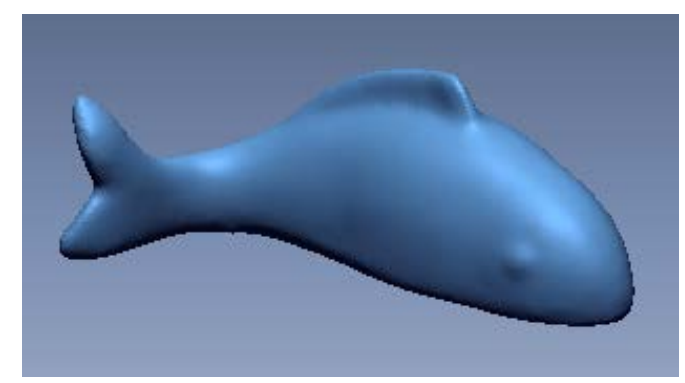

Fig. 2 point cloud data of fish 

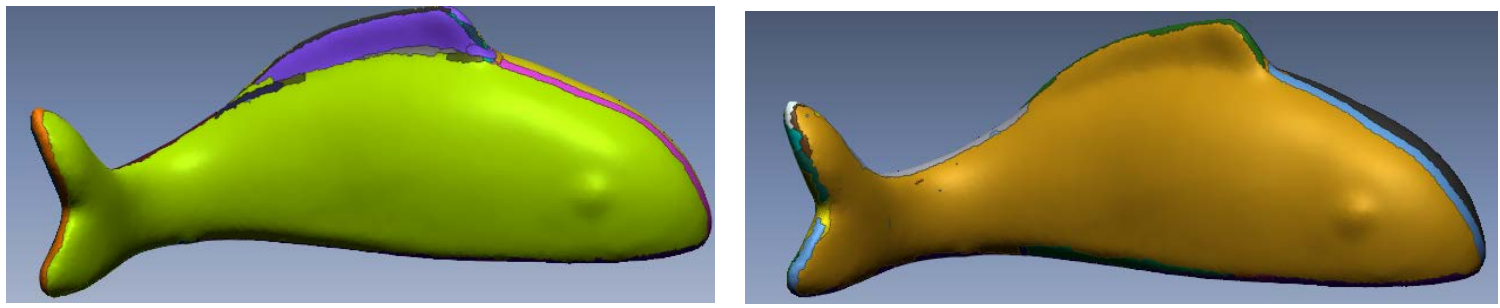

Fig. 3 two division plans of region
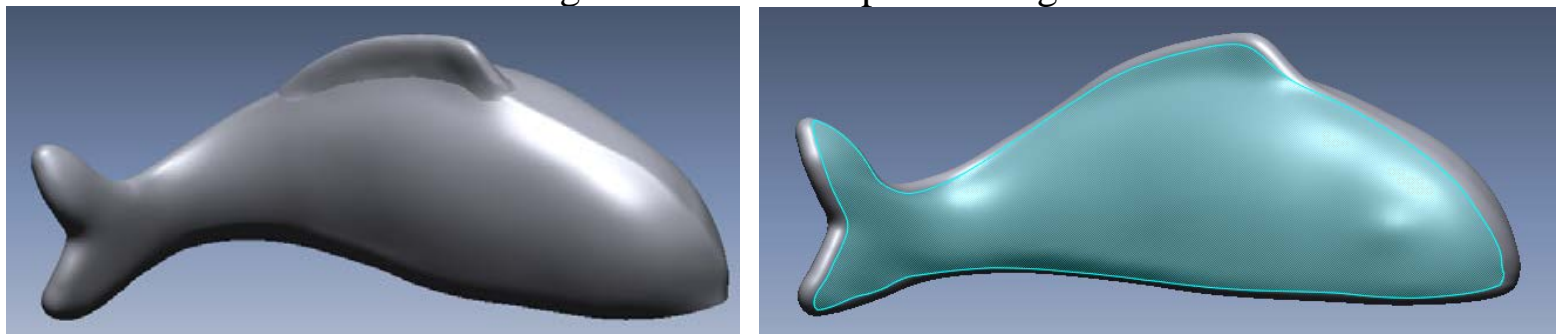

Fig. 4 the results of mesh fitting

As the example shown in Figure 5, the connecting rod model is needed to be reconstruction. The division of region is shown in Figure 6. The surface fitting by region 1 is shown in Figure 7, we can find that surfaces can't be cut at the marked place because of there isn't intersection. But, the surface fitting by region 1 and region 2, which is shown in Figure 8, is good enough to be cut, because the surfaces are intersected at the marked place. By this example, we can see that the boundary of the region is important, it can influence the shape of the surface fitted. We need to ensure the adjacent surfaces are intersected, can be cut each other.

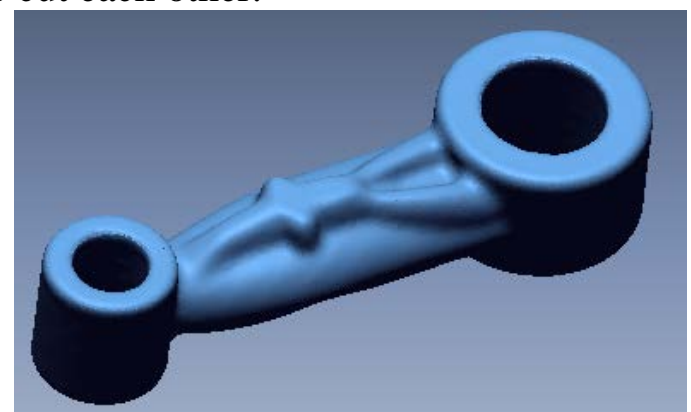

Fig. 5 point cloud data of connecting rod

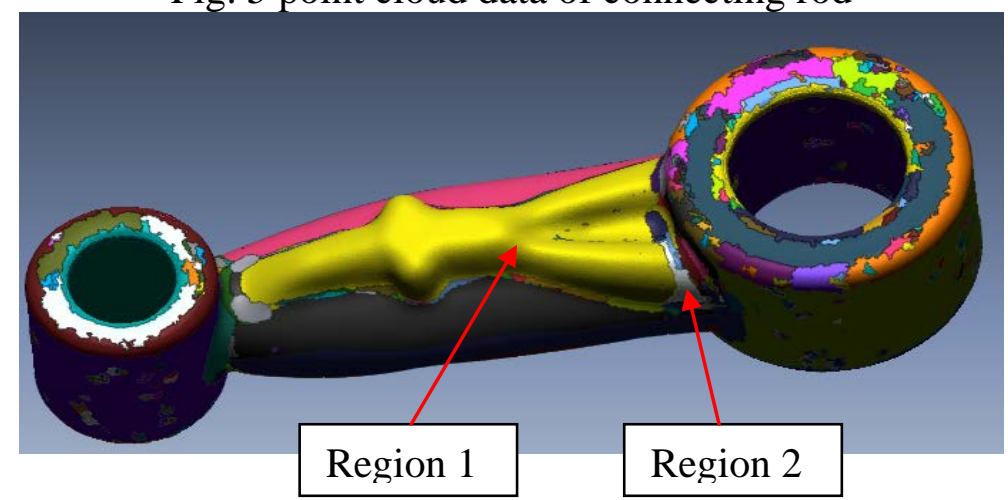

Fig. 6 division of region

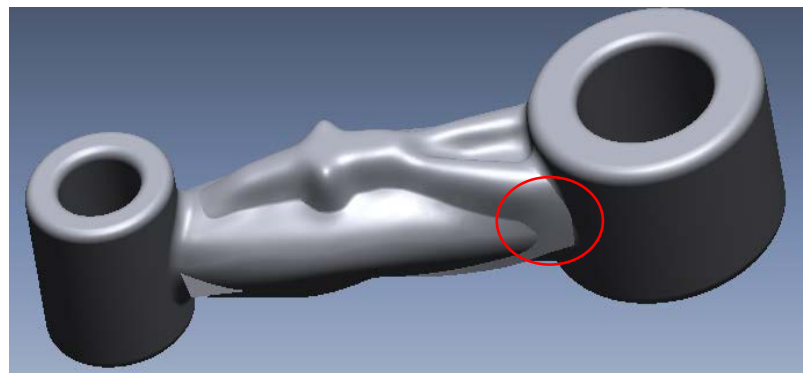

Fig. 7 fitting surface (not intersected)

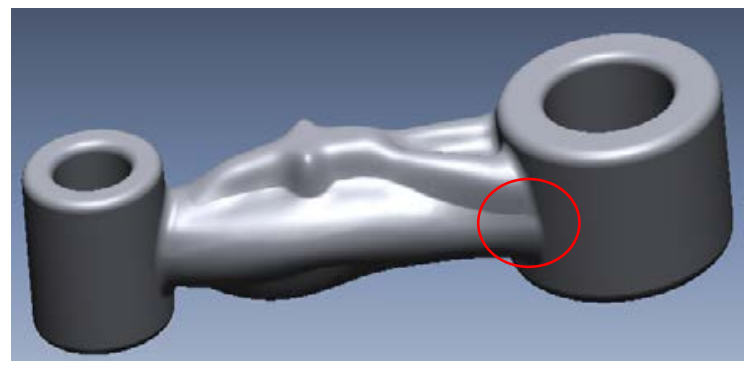

Fig. 8 fitting surface (intersected) 
As the example shown in Figure 9, the base of the massager model is needed to be reconstruction. The accuracy of the surface (shown in Figure 10) fitted by one region is not good. The accuracy of the surface (shown in Figure 11) fitted by two regions is good. By this example, we can see that the surface with large curvature can't fit by one region, we need to divide the region and fit surfaces partly, then transit the surfaces by loft.

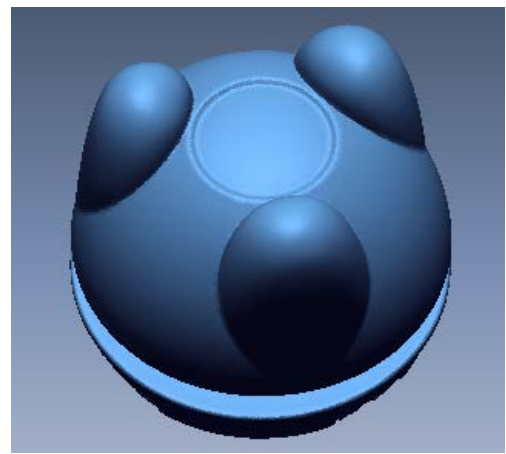

Fig. 9 point cloud data of the base of massager
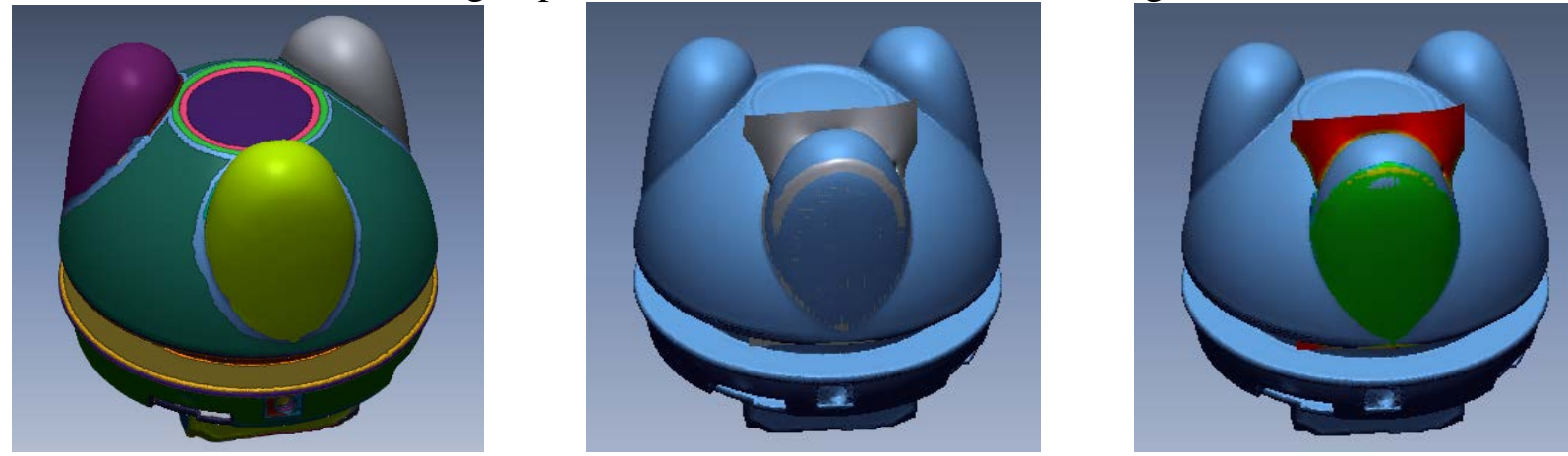

Fig. 10 fitting surface ( one region)
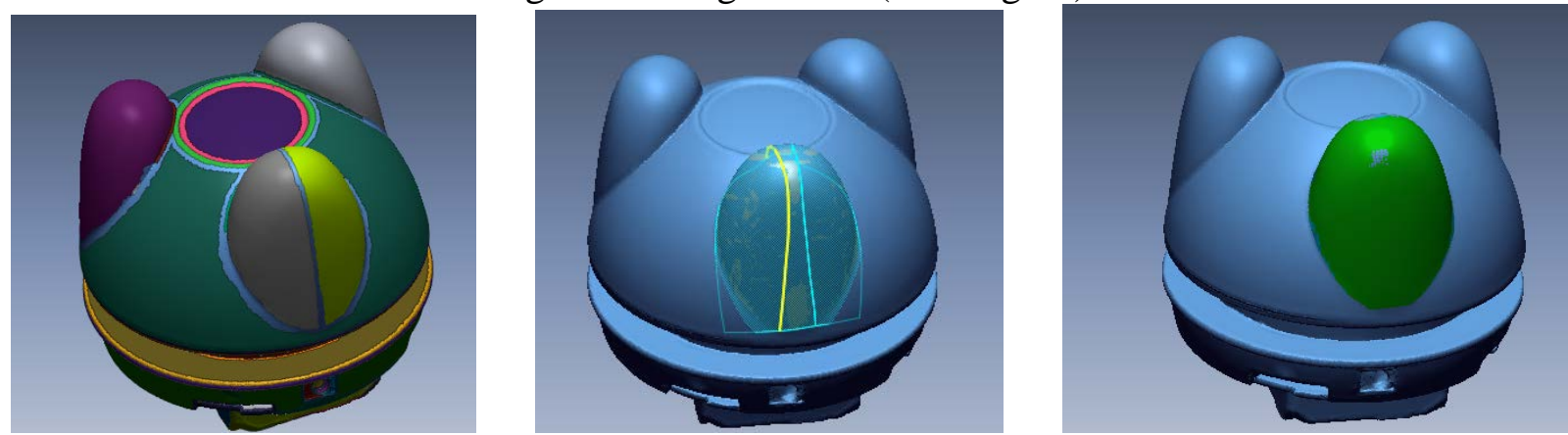

Fig. 11 fitting surface ( two regions and transition)

\section{Summary}

Division of region is important for mesh fitting, we need to divide the region by curvature, reduce the number of regions below the premise that assures accuracy, ensure the adjacent surfaces are intersected, can be cut each other.

\section{References}

[1] Information on https://zhidao.baidu.com/question/358805186.html

[2] Lin Jianli, Tang Lei, and Yong Junhai. Constuction of Non-manifold Closed Regular Triangle Mesh from Polygonal Mesh [J]. Journal of Computer-Aided Design \& Computer Graphics, 2014(26):1558-1566.

[3] CONG Hai-chen, CHENG Si-yuan, YANG Xue-rong, ZHANG Xiang-wei. Reverse Parametric Modeling Based on Division of Region [J]. Modular Machine Tool \& Automatic Manufacturing Technique, 2016(6):71-74. 
[4] Sui Yixi. Study on the Abstracting of Curve and Surface in Reverse Engineering[D]. Hang Zhou: Zhejiang University, 2008.

[5] Chang K, Chen C. 3D shape engineering and design parameterization[J]. Computer-Aided Design, 2011,5 (8 ) :681-692

[6] HUI Yan-bo, FENG Wen-yan, FENG Lan-fang, ZHANG Kun. Research on Methods of Mesh Fitting and Boundary Fitting on the Curved Surface [J]. Mechanical Research and Application, 2016(29):92-97. 